Ext ensi on of oper at i onal regi me i $n$ hi gh- temper at ure $\mathrm{pl}$ asmas and effect of ECRH on i on ther mal transport in the LHD

\begin{tabular}{|l|l|}
\hline $\begin{array}{l}\text { jour nal or } \\
\text { publ i cat i on } \mathrm{titl} \text { e }\end{array}$ & Nucl ear Fusi on \\
\hline vol une & 57 \\
\hline number & 8 \\
\hline page range & 086029 \\
\hline year & $2017-07-$ O4 \\
\hline URL & ht t p: //hdl . handl e. net /10655/00012775 \\
\hline
\end{tabular}




\title{
Extension of Operational Regime in High-Temperature Plasmas and Effect of ECRH on Ion Thermal Transport in the LHD
}

\author{
H. Takahashi ${ }^{1,2}$, K. Nagaoka ${ }^{1,3}$, S. Murakami ${ }^{4}$, M. Osakabe ${ }^{1,2}$, H. Nakano ${ }^{1}$, K. Ida ${ }^{1,2}$, \\ T. I. Tsujimura ${ }^{1}$, S. Kubo ${ }^{1,5}$, T. Kobayashi ${ }^{1,2}$, K. Tanaka ${ }^{1}$, R. Seki ${ }^{1,2}$, Y. Takeiri ${ }^{1,2}$, \\ M. Yokoyama ${ }^{1,2}$, S. Maeta ${ }^{4}$, M. Nakata ${ }^{1,2}$, M. Yoshinuma ${ }^{1,2}$, I. Yamada ${ }^{1}$, R. Yasuhara ${ }^{1}$, \\ T. Ido ${ }^{1,5}$, A. Shimizu ${ }^{1}$, H. Tsuchiya ${ }^{1}$, T. Tokuzawa ${ }^{1}$, M. Goto ${ }^{1,2}$, T. Oishi ${ }^{1,2}$, S. Morita ${ }^{1,2}$, \\ C. Suzuki ${ }^{1}$, M. Emoto ${ }^{1}$, K. Tsumori ${ }^{1,2}$, K. Ikeda $^{1}$, M. Kisaki ${ }^{1}$, T. Shimozuma ${ }^{1}$, Y. Yoshimura ${ }^{1}$, \\ H. Igami ${ }^{1}$, R. Makino ${ }^{1}$, T. Seki ${ }^{1}$, H. Kasahara ${ }^{1}$, K. Saito ${ }^{1}$, S. Kamio ${ }^{1}$, K. Nagasaki ${ }^{6}$, \\ T. Mutoh $^{7}$, O. Kaneko ${ }^{8}$, T. Morisaki ${ }^{1,2}$ and the LHD Experiment Group ${ }^{1}$
}

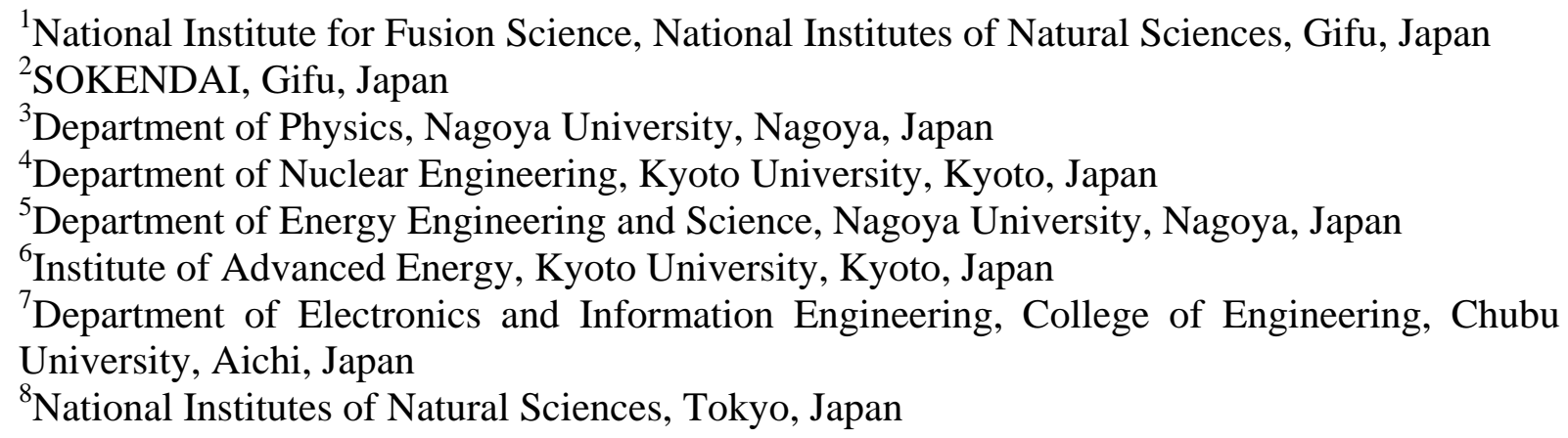

E-mail contact of main author: takahashi.hiromi@lhd.nifs.ac.jp

\begin{abstract}
Simultaneous high ion temperature $\left(T_{\mathrm{i}}\right)$ and high electron temperature $\left(T_{\mathrm{e}}\right)$ regime was successfully extended due to the optimized heating scenario in the LHD. Such high-temperature plasmas were realized by the simultaneous formation of an electron internal transport barrier (ITB) and an ion ITB by the combination of high power NBI and ECRH. Although the ion thermal confinement was degraded in the plasma core with increase of $T_{\mathrm{e}} / T_{\mathrm{i}}$ by the on-axis ECRH, it was found that the ion thermal confinement was improved at the plasma edge. The normalized ion thermal diffusivity $\chi_{\mathrm{i}} / T_{\mathrm{i}}^{1.5}$ at the plasma edge was reduced by $70 \%$. The improvement of the ion thermal confinement at the edge led to increase in $T_{\mathrm{i}}$ in the entire plasma region even though the core transport was degraded.
\end{abstract}

\section{Introduction}

Confinement improvement is necessary for realization of high-temperature plasmas and is one of the most important issues in toroidal devices. Since the first observation of $\mathrm{H}$ mode [1], various kinds of improved confinement modes have been observed in tokamaks and helical/stellarator devices. Of these improved confinement modes, an internal-transport barrier (ITB) is characterized by a steep gradient formation in temperature profiles and a decrease in thermal diffusivity in a plasma core region [2-4].

In future reactors, the fusion reaction is expected to be sustained under the electron heating dominant condition, where both the ion temperature $\left(T_{\mathrm{i}}\right)$ and the electron temperature $\left(T_{\mathrm{e}}\right)$ are high. Thus not only the investigation of the confinement improvement but also the characterization of the thermal transport for the plasmas, of which $T_{\mathrm{i}}$ and $T_{\mathrm{e}}$ are simultaneously high, are necessary. In the present status, such a plasma condition is realized by the combination heating of a neutral beam injection (NBI) and an electron cyclotron 
resonance heating (ECRH). The effect of a $T_{\mathrm{e}} / T_{\mathrm{i}}$ and/or an ECRH on the ion thermal confinement has been studied in several devices [5-9].

In the Large Helical Device (LHD), electron-ITB (e-ITB) relating to the production of high $T_{\mathrm{e}}$ plasmas has been realized by strongly centre-focused ECRH beams [10-13]. The radial electric field $E_{r}$ was measured by charge exchange recombination spectroscopy (CXRS) [14] and/or the heavy ion beam probe (HIBP) [15]. The formation of the strong positive $E_{r}$ was observed in the core region [10], which well agreed with the prediction of the neoclassical transport theory, and the electron thermal confinement improved by a factor of 8 inside the ITB. An e-ITB have been observed in other helical devices as summarized in Ref. [16] and the interest has renewed since beginning of an operation of the Wendelstein 7-X, with Core Electron Root Plasmas [17]. High- $T_{\mathrm{i}}$ accompanied with an ion-ITB (i-ITB) has been achieved in NBI-sustained plasmas in combination with a carbon pellet [18-22]. The gradient of $T_{\mathrm{i}}$ at the core region increased and the ion thermal diffusivity was clearly reduced. The i-ITB observed in the LHD is characterized by centre-peaked $T_{\mathrm{i}}$ profile in contrast with a so-called box-type ITB in tokamaks [2, 23]. The momentum transport was found to be improved and the strong-peaked profiles of the toroidal flow velocity were formed [21, 22]. The formation of weak negative $E_{r}$ in the core and the strong positive $E_{r}$ in the peripheral region were also found [21].

In the previous experiments above, an i-ITB and an e-ITB have been obtained independently [22], indicating decoupling with each other, which is different from tokamak ITBs. Therefore i-ITB and e-ITB had been studied individually. In recent years, an integration of high $T_{\mathrm{i}}$ and high $T_{\mathrm{e}}$ with the simultaneous formation of an i-ITB and an e-ITB has been successfully achieved in the LHD by the combination of the NBI and the ECRH [8].

The present paper shows the high-temperature plasma regime extended recently and the effect of an ECRH on the ion thermal transport in the LHD and is organized as follows. In section 2, the experimental setup is described. Section 3 treats the extension of the operational regime in high temperature plasmas in the LHD. The temperature profiles are also compared among the four different confinement states. Section 4 focuses on the effect of the ECRH on $T_{\text {i }}$ profile. The response of the $T_{\mathrm{i}}$ gradients to the ECRH and the dependence of the change in the ion temperature profile during the ECRH on the electron density $\left(n_{\mathrm{e}}\right)$ are described. Section 5 is discussion. The effect of the ECRH on the the ion thermal diffusivity and the response of the density fluctuation to the ECRH are shown. The summary is in section 6 .

\section{Experimental Setup}

The LHD is the largest heliotron device with a majour radius $R=3.9 \mathrm{~m}$, a minor radius $a=0.6 \mathrm{~m}$, a pole number of 2 and a toroidal period of 10 [24, 25]. The heliotron configurations are produced by a set of helical winding coils and three sets of poloidal field coils, which are all superconducting magnets. The magnetic field on the axis is $\sim 2.9 \mathrm{~T}$. In the LHD, three negative-ion-based NBIs produce hydrogen neutral beams with the beam energy of $180 \mathrm{keV}$ and total port-through power of $16 \mathrm{MW}$ [26]. The negative-NBIs are tangentially injected into LHD plasmas. The positive-ion-based NBIs with low energy of $40 \mathrm{keV}$ were perpendicularly injected for ion heating [27]. In 2010, second perpendicular-NBI

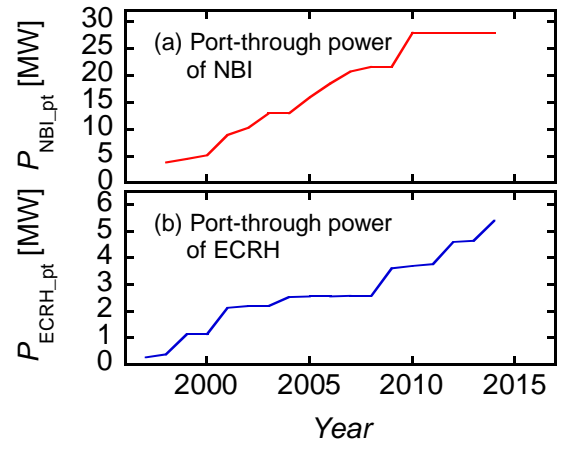

FIG. 1. The history of the port-through power of (a) the NBI and (b) the ECRH in the LHD. 
with the beam energy of $40 \mathrm{keV}$ was installed in the LHD and the total-port through power of perpendicular-NBIs reached $12 \mathrm{MW}$. An ECRH system with eight gyrotrons has been operated for preionization and plasma heating [28]. Of these, the high power 77-GHz gyrotrons with the output power of more than $1 \mathrm{MW}$ have been operated since the experimental campaign in 2007 [28, 29]. In the present state, three $77 \mathrm{GHz}$ gyrotrons are operational for plasma experiments. In addition to these $77 \mathrm{GHz}$ tubes, two high power gyrotrons (154 GHz, $1 \mathrm{MW}$ ) were installed in FY2012 and FY2014 for high density plasma heating [13]. The configuration of the ECRH transmission line has also been changed to enhance the central heating capability. In the present condition, four of the five high power ECRH antennas are set at the horizontal ports, then the deeper injection angle of the beams and the smaller beam refraction compared with the injection from the vertical port have been realized. The heating capability on the LHD has been upgraded year by year. Figure 1 shows the history of the port-through power of (a) the NBI and (b) the ECRH in the LHD. The total injection power of the NBI and the ECRH reached $28 \mathrm{MW}$ and 5.4 MW, respectively.

\section{Extension of High Temperature Regime}

\subsection{Response of Temperature Gradients to ECRH}

Figure 2 shows (a) the latest high-temperature operational regime in the LHD, and (b) the radial profiles of $T_{\mathrm{i}}, T_{\mathrm{e}}$, and $n_{\mathrm{e}}$ for a typical high-temperature plasma associated with the eITB and the i-ITB. The variable $r_{\text {eff }} / a_{99}$ in the horizontal axis of Fig. 2 (b) is the effective minor radius normalized by the averaged minor radius where $99 \%$ of the electron stored energy is confined. We finely adjusted the ECRH injection angle and the EC wave polarization taking account of the actual plasma profiles in real time [30]. In Fig. 2 (a), the gray area was obtained using only NBI or ECRH, the blue area was using both 26-MW NBI

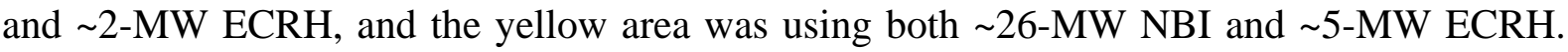
The circles and the squares represent the data with and without the adjustment, respectively. The operational regime was successfully extended mainly due to the upgraded ECRH system and the optimization of the ECRH injection. The global parameters of the discharge of Fig. 2 (b) are: the line-averaged electron density measured using FIR $n_{\mathrm{e} \text { f fir }}$ was $1.2 \times 10^{19} \mathrm{~m}^{-3}$; the energy confinement time $\tau_{\mathrm{E}}$ was $33 \mathrm{~ms}$; and the volume-averaged beta value $<\beta>$ was $0.53 \%$, where the kinetic energy was used for the evaluation of $\tau_{\mathrm{E}}$ and $\langle\beta\rangle$. Although the $T_{\mathrm{i}}$ profile was flattened in the central region due to the confinement degradation by increase in $T_{\mathrm{e}} / T_{\mathrm{i}}$, the i-ITB were maintained (actually, $d T_{\mathrm{i}} / d r_{\mathrm{eff}}$ at $r_{\mathrm{eff}} / a_{99}=0.6$ was 2.5 times larger than that of $\mathrm{L}$ mode plasma).

\subsection{Comparison of the Response of Temperature Gradients to ECRH}

The systematic data of the temperature profiles have been obtained for several confinement states in the LHD. Figure 3 shows the comparison of the temperature, electron density, and the temperature gradient profiles among four different confinement states, (a), (b) the L mode plasma, (c), (d) the iITB plasma, (e), (f) the e-ITB plasma, and (g), (h) the $\mathrm{i}-$ and e-ITB plasma. The magnetic configuration is the
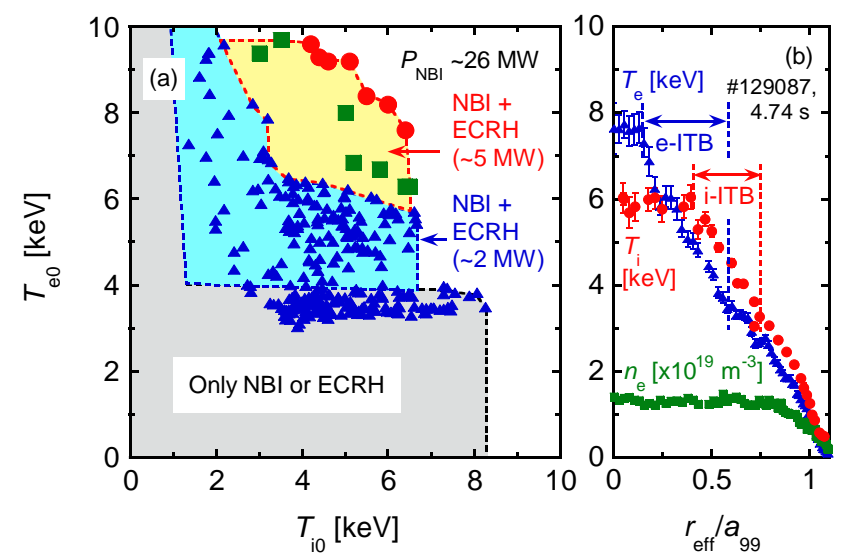

FIG. 2. (a) Extended operational high-temperature regime in the LHD, (b) $T_{\mathrm{i}}, T_{\mathrm{e}}$, and $n_{\mathrm{e}}$ profiles for a typical high-temperature plasma with the ITBs. 

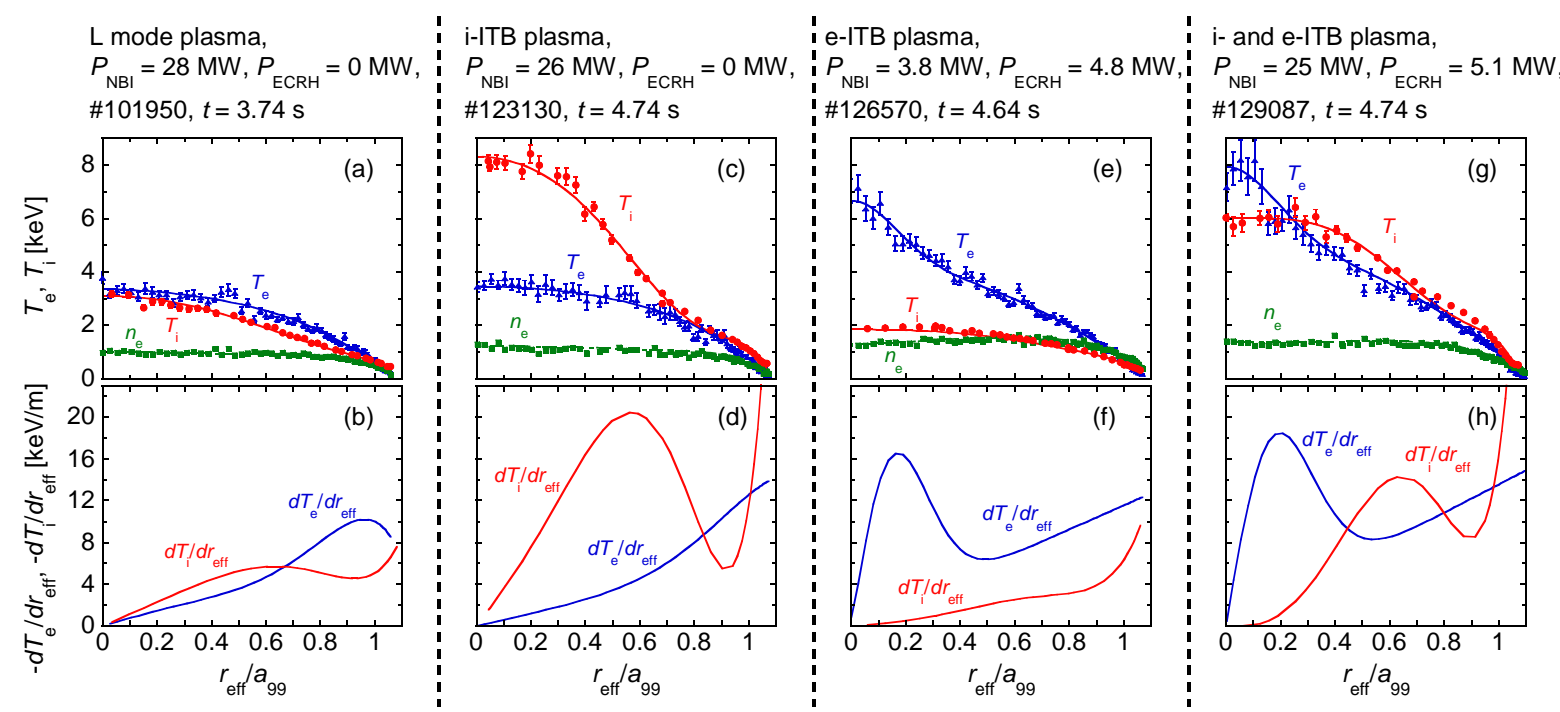

FIG. 3. The comparison of the temperature and the gradient profiles among four different confinement states, (a), (b) the L mode plasma, (c), (d) the i-ITB plasma, (e), (f) the e-ITB plasma, and (g), (h) the i- and e-ITB plasma.

vacuum-magnetic axis $R_{\mathrm{ax}}$ of $3.6 \mathrm{~m}$ and the toroidal magnetic field on the axis $B_{t}$ of 2.75-2.85 T. The L mode plasma and the i-ITB plasma were produced using NBI alone (26-28 MW). The e-ITB plasma was mainly heated using the on-axis ECRH (4.8 MW). In addition to the ECRH, a diagnostic NBI (3.8 MW) was injected in order to measure the $T_{\mathrm{i}}$ profile by CXRS. The i- and e-ITB plasma was obtained using both the NBI (25 MW) and the on-axis ECRH (5.1 MW). The $n_{\mathrm{e}}$ profiles were flat except for the e-ITB plasma. The $n_{\mathrm{e}}$ profile of the e-ITB plasma was a slightly hollow shape due to the smaller core fuelling of the NBI compared with the other three cases and the density clamping effect by the on-axis ECRH. The line-averaged density $n_{\mathrm{e}-\text { fir }}$ was $0.87 \times 10^{19} \mathrm{~m}^{-3}$ for the L mode plasma, $1.0 \times 10^{19} \mathrm{~m}^{-3}$ for the i-ITB plasma, 1.3 $\mathrm{x} 10^{19} \mathrm{~m}^{-3}$ for the e-ITB plasma, and $1.2 \times 10^{19} \mathrm{~m}^{-3}$ for the i- and e-ITB plasma. Here we focus on the description of the profile characteristics of the i- and e-ITB plasma. Please see the references for the details of the confinement characteristics for the i-ITB [18-22] and the eITB [10-13] in the LHD.

For the i- and e-ITB plasma, the ion-ITB structure was maintained in the plasma core region even the peak value of the $d T_{\mathrm{i}} / d r_{\text {eff }}$ at $r_{\text {eff }} / a_{99} \sim 0.6$ was slightly degraded compared with i-ITB plasma. The $T_{\text {i }}$ profile became almost flattened in the plasma central region. On the other hand, the $d T_{\mathrm{i}} / d r_{\text {eff }}$ greatly increased in the plasma edge. Compared with L mode plasma, the $d T_{\mathrm{e}} / d r_{\text {eff }}$ was significantly increased in the core region due to the formation of the e-ITB. The shape of the profiles, and especially the position of the local maximum and the local minimum of the $d T_{\mathrm{e}} / d r_{\mathrm{eff}}$ and the $d T_{\mathrm{i}} / d r_{\mathrm{eff}}$ are approximately the same as the $d T_{\mathrm{e}} / d r_{\text {eff }}$ of the eITB plasma and the $d T_{\mathrm{i}} / d r_{\text {eff }}$ of the i-ITB, respectively. The ITB positions were different between the $T_{\mathrm{i}}$ and the $T_{\mathrm{e}}$, namely the e-ITB was formed at the inner position compared with i-ITB. The difference of the ITB positions has been observed in the LHD [8]. As a result, the $T_{\mathrm{e}} / T_{\mathrm{i}}$ tends to be higher in the central region for i- and e-ITB plasmas.

\section{Effect of ECRH on Ion Temperature Profile}

\subsection{Response of Temperature Gradients to ECRH}

Simultaneous high $T_{\mathrm{i}}$ and high $T_{\mathrm{e}}$ have been obtained by the on-axis ECRH superposition on the high- $T_{\mathrm{i}}$ plasmas sustained by the NBI. In such condition, a seesaw-like behavior of the ion 
thermal transport between core and edge has been observed. As shown in Fig. 3 (g), the $T_{\mathrm{i}}$ gradient was decreased in the plasma core due to the superposition of the on-axis ECRH. On the other hand, the $T_{\mathrm{i}}$ gradient increased in the plasma edge. Here we discuss the details of the effect of the ECRH on the $T_{\text {i }}$ profile.

Figure 4 shows the time evolution of (a) the port through power of the NBI and the ECRH, (b) the line-averaged-electron density, (c), (d) the gradient of $T_{\mathrm{e}}$ and $T_{\mathrm{i}}$ at $r_{\mathrm{eff}} / a_{99}=0.31$ and 0.98 , and (e) the kinetic energy for electron and ion. The on-axis ECRH was superposed stepwise up to $5 \mathrm{MW}$ on the NBI-sustained plasma. The ray-tracing calculation showed that more than $90 \%$ of the ECRH power was deposited inside $r_{\text {eff }} / a_{99}=0.2$ at $4.74 \mathrm{~s}$. Both in the plasma core and the edge, $T_{\mathrm{e}}$ and the gradient increased with increase in the ECRH power. On the other hand, the gradient of $T_{\mathrm{i}}$ was degraded in the plasma core with increase in the ECRH power. In contrast, the $T_{\mathrm{i}}$ gradient was improved at the plasma edge. We confirmed that the ion heating power of the NBI increased during the ECRH superposition because of the increase in the beam critical energy by $T_{\mathrm{e}}$ increase. However, the increment of the ion heating power was $11 \%$ from the comparison between $t=4.24 \mathrm{~s}$ and $4.74 \mathrm{~s}$, and this cannot explain the doubled $d T_{\mathrm{i}} / d r_{\text {eff }}$ at the edge. This edge gradient increase is considered to be a different phenomenon from the so-called confinement transition because the edge gradient changed linearly with the step-by-stepincreased ECRH power. The ion kinetic energy was also increased with the increase in the ECRH power due to the steepened $T_{\mathrm{i}}$ gradient at the edge.

\subsection{Dependence of Change in Temperature Profiles during ECRH on Electron Density}

As introduced in Sec. 4.1, temperature profiles are clearly affected by the ECRH not only for the electron but also for the ion. Here the temperature profile modification by the ECRH and the dependence on $n_{\mathrm{e}}$ range are introduced. Figure 5 shows the radial temperature profiles of (a) electron and (b) ion before and during the ECRH (5.1 MW), and (c) the time evolution of the relation between $T_{\mathrm{e}}$ and $T_{\mathrm{i}}$ at several radial positions during the ECRH power changing for lower line-averaged $n_{\mathrm{e}}$ of $1.0 \times 10^{19} \mathrm{~m}^{-3}$, and (d)-(f) for higher line-averaged $n_{\mathrm{e}}$ of $1.4 \times 10^{19} \mathrm{~m}^{-3}$. The dashed lines in Fig. 5 (c), (f) represent $T_{\mathrm{e}} / T_{\mathrm{i}}=1$. In both discharges, five ECRH beams of $1 \mathrm{MW}$ each were superposed in stepwise on the NBI-sustained plasmas as shown in Fig. 4 (a) and were focused on the axis.

For the lower $n_{\mathrm{e}}$ case, $T_{\mathrm{e}}$ and the gradients increased in the entire plasma region, and especially in the core due to the ITB. The increase in the gradient outside of the ITB was due to the increase in the electron heat flux by the ECRH superposition. On the other hand, a seesaw-like behaviour was observed in the $T_{\mathrm{i}}$ profile. Here $T_{\mathrm{e}} / T_{\mathrm{i}}$ exceeded 1 in the core region by the centre-focused ECRH, then $T_{\mathrm{i}}$ and its gradient degraded, resulting in the largely flattened $T_{\mathrm{i}}$ profile. In contrast, the $d T_{\mathrm{i}} / d r$ increased in the edge, leading to the $T_{\mathrm{i}}$ increase in the wide region except for the plasma centre. For the higher $n_{\mathrm{e}}$ case, the responses of $T_{\mathrm{e}}$ and $T_{\mathrm{i}}$ 
Lower $n_{\mathrm{e}}$ case, $n_{\mathrm{e}} \sim 1.0 \times 10^{19} \mathrm{~m}^{-3}$
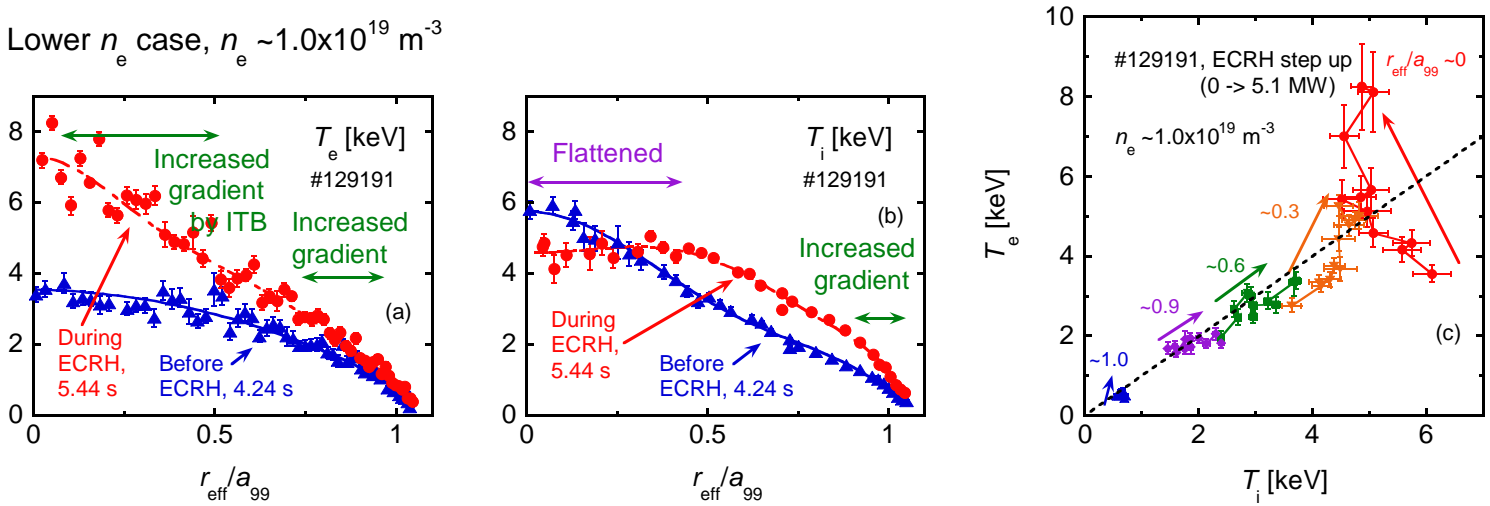

Higher $n_{\mathrm{e}}$ case, $n_{\mathrm{e}} \sim 1.4 \times 10^{19} \mathrm{~m}^{-3}$
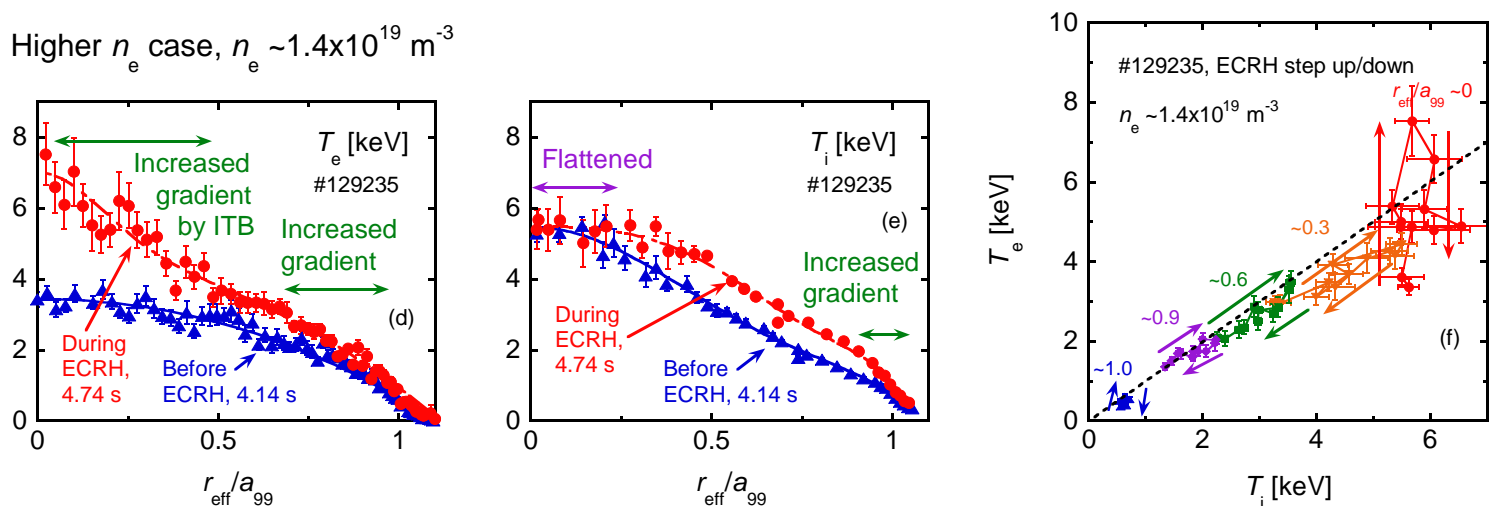

FIG. 5. The radial temperature profiles of (a) electron and (b) ion before and during the ECRH (5.1 $\mathrm{MW}$ ), and (c) the time evolution of the relation between $T_{\mathrm{e}}$ and $T_{\mathrm{i}}$ at several radial positions during the ECRH power changing for lower line-averaged $n_{\mathrm{e}}$ of $1.0 \times 10^{19} \mathrm{~m}^{-3}$, and (d)-(f) for higher lineaveraged $n_{\mathrm{e}}$ of $1.4 \times 10^{19} \mathrm{~m}^{-3}$.

profiles to the ECRH were similar to lower $n_{\mathrm{e}}$ situation but the decrease in $T_{\mathrm{i}}$ was not observed in the plasma centre. In the study, the core $T_{\mathrm{i}}$ was found to be determined by the competition between core-confinement degradation and the edgeconfinement improvement. Although the $d T_{\mathrm{i}} / d r$ around the centre degraded, $T_{\mathrm{i}}$ itself was not decreased even though $T_{\mathrm{e}} / T_{\mathrm{i}}$ exceeded 1 due to (1) the improvement of peripheral $T_{\mathrm{i}}$ gradient and (2) the i-ITB was maintained in the more inward side compared with lower $n_{\mathrm{e}}$ by the decrease of the flattening area.

Figure 6 shows the radial profiles of the increment in $T_{\mathrm{i}}$ by the ECRH superposition for two $n_{\mathrm{e}}$ cases.

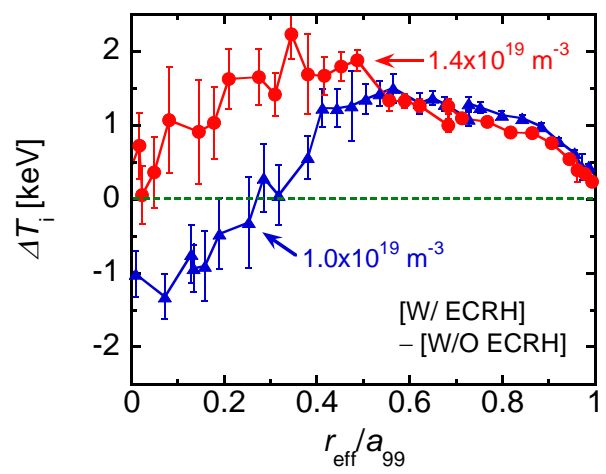

FIG. 6. The radial profiles of the increment in $T_{\mathrm{i}}$ by ECRH superposition for two $n_{\mathrm{e}}$ cases.

These are obtained as subtractions of data in Fig. 5 (b) and (e), respectively. The $T_{\mathrm{i}}$ increment in the inner half of the minor radius were different between the two conditions. Core $T_{\mathrm{i}}$ can be increased by the on-axis ECRH in higher $n_{\mathrm{e}}$ plasmas. This is a beneficial feature for a high $T_{\mathrm{i}}$ scenario for high density plasmas under electron heating dominant like DEMO. The reason has not been clarified why the higher $n_{\mathrm{e}}$ was better to sustain higher core $T_{\mathrm{i}}$ in the high $T_{\mathrm{e}} / T_{\mathrm{i}}$ situation. Actually the equipartition heating power was -0.1 MW (minus means the heat flux moved to electron from ion) on the other hand the absorbed-ion-heating power of the NBI was 7.0 MW for $n_{\mathrm{e}}=1.4 \times 10^{19} \mathrm{~m}^{-3}$ case. Thus the contribution of the equipartition heat flux is 
considerably small. The systematic experiment with the various $n_{\mathrm{e}}$ conditions is required to explain the results.

\section{Discussion}

\subsection{Change in Ion Thermal Diffusivity by ECRH}

In the previous section,
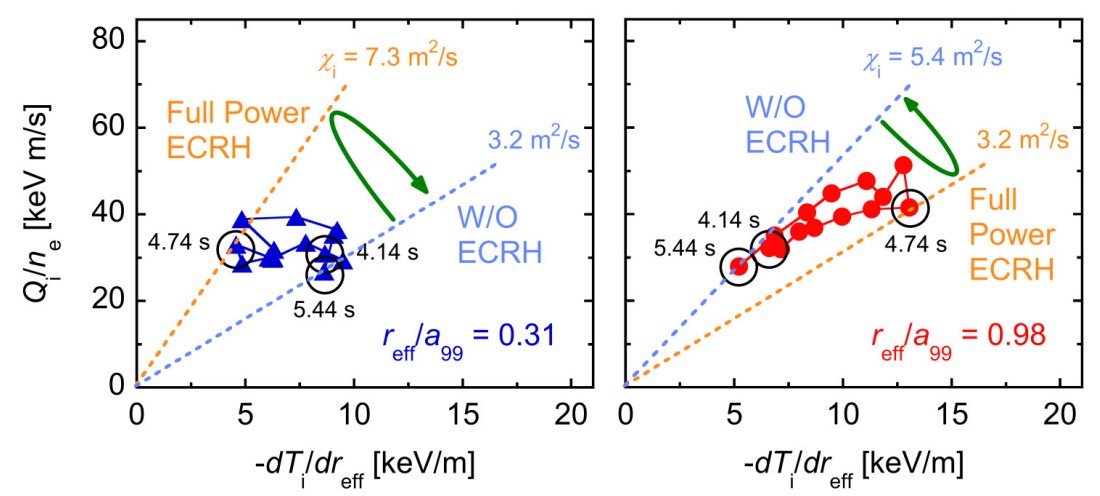

FIG. 7. The flux-gradient relation between $Q_{\mathrm{i}} / n_{\mathrm{e}}$ and $d T_{\mathrm{i}} / d r_{\text {eff }}$ at (a) $r_{\text {eff }} / a_{99}=0.31$ and (b) 0.98 . the modification of the ion temperature profile by the ECRH was discussed. Here the temporal change in the ion thermal confinement during the ECRH superposition in the present discharge is treated. Dynamic transport analysis taking into account the slowing-down effect of the NBI deposition is suitable when the plasma parameters change transiently in the discharge [31]. Figure 7 shows the flux-gradient relation between $Q_{\mathrm{i}} / n_{\mathrm{e}}$ and $d T_{\mathrm{i}} / d r_{\text {eff }}$ at (a) $r_{\text {eff }} / a_{99}=0.31$ and (b) 0.98, where $Q_{\mathrm{i}}$ is the ion-heat flux. The slope in the relation between $Q_{\mathrm{i}} / n_{\mathrm{e}}$ and $d T_{\mathrm{i}} / d r_{\text {eff }}$ correspond to $\chi_{\mathrm{i}}$. The target discharge is that shown in Fig. 4, namely the on-axis ECRH of 1-MW each was superposed in stepwise up to $5.1 \mathrm{MW}$ on the plasma sustained by the 25-MW NBI. Two branches of $\chi_{\mathrm{i}}$ are illustrated in each figure. One is the branch at the timing of no ECRH superposition (25-MW NBI alone) and another is that of full ECRH superposition (both 5.1MW ECRH and 25-MW NBI). As shown in Fig. 7 (a), $d T_{\mathrm{i}} / d r_{\text {eff }}$ became half with the increase in the power of the ECRH superposition despite the small change of $Q_{\mathrm{i}} / n_{\mathrm{e}}$ at $r_{\mathrm{eff}} / a_{99}=0.31$. This indicates that the ion-thermal confinement was degraded. Then the ion thermal confinement returned to the original confinement state with the decrease in the ECRH power. On the other hand, the ion thermal confinement at the edge improved due to the on-axis ECRH and the dependence of the ion thermal confinement on the ECRH power was opposite to that at the core region.
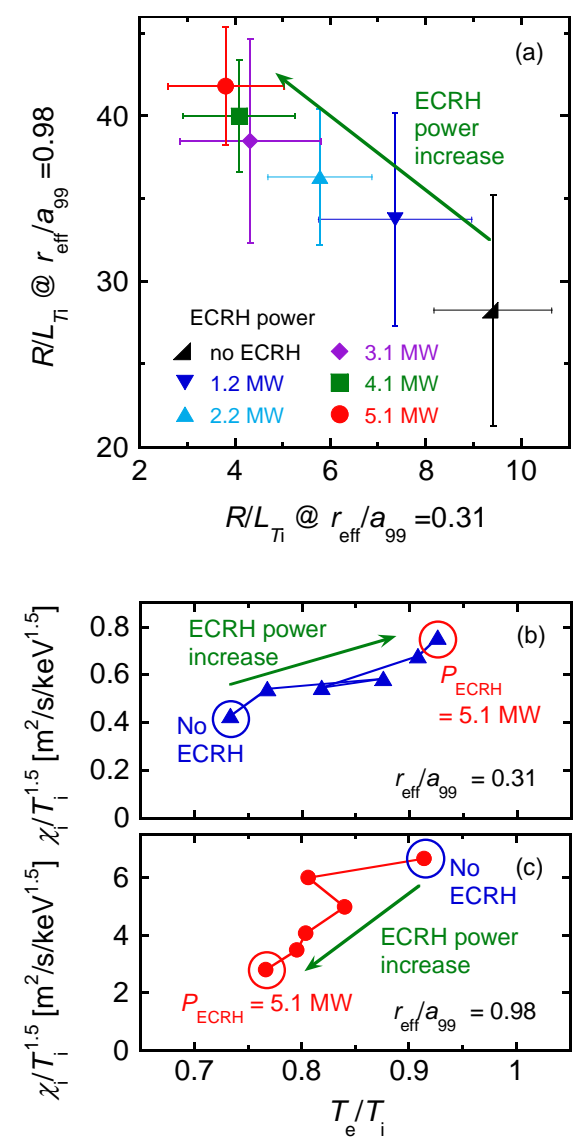

FIG. 8. (a) The relation of the normalized scale length of the ion temperature gradient $R / L_{T \mathrm{1}}$ between the core and the edge, the ion thermal diffusivity normalized by the GyroBohm factor $\chi_{\mathrm{i}} / T_{\mathrm{i}}^{1.5}$ at (b) the core and (c) the edge.

Figure 8 shows (a) the relation of the normalized scale length of the ion temperature gradient $R / L_{T 1}$ between the core and the edge, the ion thermal diffusivity normalized by the GyroBohm factor $\chi_{\mathrm{i}} / T_{\mathrm{i}}^{1.5}$ at (b) the core, and (c) the edge. The ion thermal diffusivity was evaluated by a power balance analysis. $R / L_{T 1}$ degraded by $56 \%$ at the core but improved by $50 \%$ at the edge. The normalized ion thermal diffusivity was small when the $T_{\mathrm{e}} / T_{\mathrm{i}}$ was small both at the core and the edge even though the dependence on $P_{\mathrm{ECRH}}$ was opposite. The 
causality of the change in the ion thermal transport is supposed to be different between the core and the edge. In the plasma central region, $T_{\mathrm{e}}$ increased by the ECRH, then $T_{\mathrm{e}} / T_{\mathrm{i}}$ increased and the ion thermal transport was considered to be degraded. On the other hand, $T_{\mathrm{i}}$ increased at the edge by the ECRH, accordingly, $T_{\mathrm{e}} / T_{\mathrm{i}}$ decreased. The normalized ion thermal diffusivity $\chi_{\mathrm{i}} / T_{\mathrm{i}}^{1.5}$ at $r_{\mathrm{eff}} / a_{99}=0.98$ was reduced by $70 \%$. The improvement of the ion thermal transport at the edge led to the increase in $T_{\mathrm{i}}$ in the entire plasma region and the increase in the ion kinetic energy even though the core ion thermal transport was degraded.

The ambipolar radial electric field $E_{r_{-} \text {am }}$ and the neoclassical ion thermal diffusivity $\chi_{\mathrm{i} \_\mathrm{NC}}$ were calculated using DCOM code [32]. Although the $E_{r}$ was not measured for the plasmas presented here, the formation of the negative $E_{r}$ was confirmed in the i-

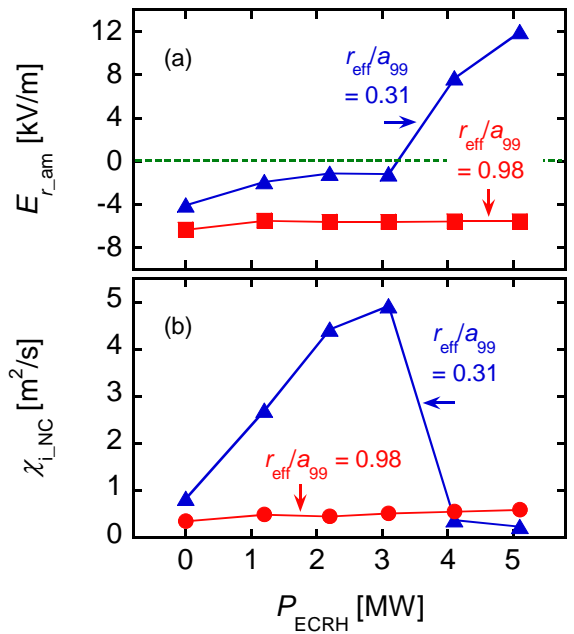

FIG. 9. The dependence of (a) the $E_{r_{-} \text {am }}$ and (b) the $\chi_{i_{-} N C}$ on the superposed ECRH power at $r_{\text {eff }} / a_{99}=0.31$ and 0.98 .

ITB plasmas [21]. Also the positive $E_{r}$ formation was found in the plasma with both e- and iITBs [8]. Figure 9 shows the dependence of (a) the $E_{r_{-} \text {am }}$ and (b) the $\chi_{\mathrm{i}_{-} \mathrm{NC}}$ on the superposed ECRH power at $r_{\text {eff }} / a_{99}=0.31$ and 0.98 . The core $E_{r_{-} \text {am }}$ changed from negative to positive with increase in the ECRH power (with increase in the $T_{\mathrm{e}} / T_{\mathrm{i}}$ ). The core $\chi_{\mathrm{i} \_ \text {NC }}$ increased up to $P_{\mathrm{ECRH}}=3 \mathrm{MW}$ due to the decrease in the negative $E_{r_{-} \text {am }}$ and was significantly decreased in $P_{\mathrm{ECRH}}>4 \mathrm{MW}$ due to the presence of the large value of the $E_{r_{-} \text {am }}$ in the electron-root. The effect of the ECRH on the edge $E_{r_{-} \text {am }}$ and the $\chi_{\text {i NCN }}$ looks minor compared with the core region. On the other hand, the experimental $\chi_{\mathrm{i}}$ from the power balance analysis increased with increase in the $T_{\mathrm{e}} / T_{\mathrm{i}}$ as shown in the Fig. 8 (b) and (c). The tendency of the $\chi_{\mathrm{i}}$ on the ECRH power was quite different between the experiment and the neoclassical calculation thus the ion thermal confinement of the plasma presented here is considered to be strongly modified by the anomalous transport depending on the $T_{\mathrm{e}} / T_{\mathrm{i}}$.

\subsection{Response of Fluctuation to ECRH}

The effect of the ECRH on the behavior of the fluctuation was also investigated. Figures 10 (a) and (b) are the time and space dependent cross correlation function [33, 34] defined as

$C(\tau, \rho)=\frac{\left\langle\tilde{n}_{\mathrm{e}}(t, \rho) \tilde{n}_{\mathrm{e}}\left(t-\tau, \rho_{0}\right)\right\rangle}{\sqrt{\left\langle\tilde{n}_{\mathrm{e}}^{2}(t, \rho)\right\rangle\left\langle\tilde{n}_{\mathrm{e}}^{2}\left(t, \rho_{0}\right)\right\rangle}}$,

where $\tilde{n}_{\mathrm{e}}$ denotes the $n_{\mathrm{e}}$ fluctuation having an intermediate frequency range $(f<20 \mathrm{kHz})$. The $n_{\mathrm{e}}$ fluctuation was measured using microwave frequency comb Doppler reflectometer [35]. The reference radius $\rho_{0}$ is taken as the outer most measurement point of the reflectometer. The angular bracket indicates the time average. The strength of the spatial correlation can be evaluated as the time average of the envelope of the cross correlation function defined as

$\frac{1}{N} \sum_{\tau}|C(\tau, \rho)|_{\mathrm{E}}$,

where $|*|_{E}$ denotes the envelope of the signal. This quantity is shown in Fig. 10 (c) as a function of the ECRH injection power. These data were obtained for the discharge introduced in Fig. 4. As can be seen from the figure, the radial structure of the edge fluctuation was 

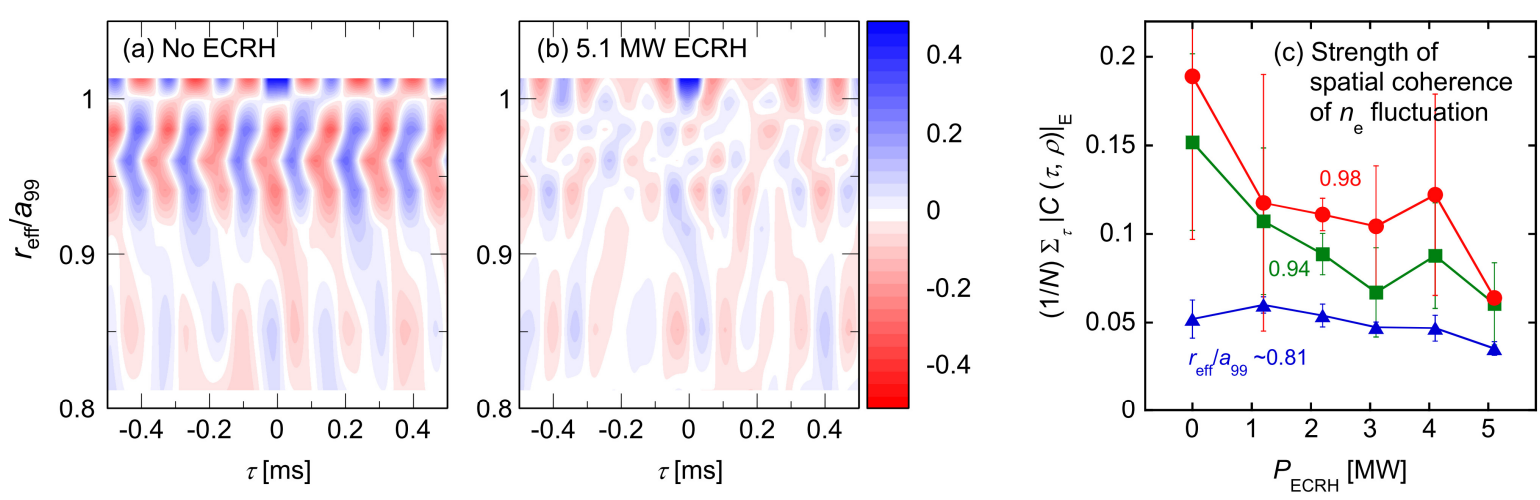

FIG. 10. The time and space dependent cross correlation function for the timing of (a) no ECRH and (b) $5.1 \mathrm{MW}$ ECRH, (c) the ECRH power dependence of the time average of the envelope of the cross correlation function.

segmentalized by the on-axis ECRH superposition. This indicates that the correlation length of the fluctuation decreased. Also, the strength of the spatial correlation decreased with increase in the ECRH power due to the change in the correlation length and the correlation time.

We also measured the $n_{\mathrm{e}}$ fluctuation using two dimensional phase contrast imaging (2-D PCI) [36]. Figure 11 shows the radial profiles of (a), (b) the phase velocity, (c), (d) the amplitude of the $n_{\mathrm{e}}$ fluctuation, and (e) $\chi_{\mathrm{i}} / T_{\mathrm{i}}^{1.5}$ before and during the ECRH (5.1 MW). The data in Fig. 11 (a) and (c) was obtained using 2-D PCI at the lower side of the equatorial plane and (b) and (d) were at the upper side. The ion thermal diffusivity was obtained by the power balance analysis. The up-down asymmetry of the fluctuation structure was observed in the experiments. This is considered due to the tilting of the turbulence eddy $[36,37]$. The phase velocity in the ion-diamagnetic direction increased in the entire plasma region by the ECRH superposition. This indicates that the positive $E_{r}$ increased. The fluctuation amplitude was increased in the inner half of the plasma radius during the ECRH superposition. Especially, the response of the fluctuation amplitude in the upper side to the ECRH coincidently correlated with the change in $\chi_{\mathrm{i}} / T_{\mathrm{i}}^{1.5}$, namely, the amplitude and the thermal diffusivity were increased in the inner half of the plasma radius and were decreased in the outer half of the radius.

\section{Summary}

Simultaneous high $T_{\mathrm{i}}$ and high $T_{\mathrm{e}}$ regime was successfully extended in the LHD. The integration of high $T_{\mathrm{i}}$ and high $T_{\mathrm{e}}$ was realized due to the establishment of the i-ITB and e-ITB simultaneously by the NBI and the ECRH mix. It was found that the ion thermal confinement
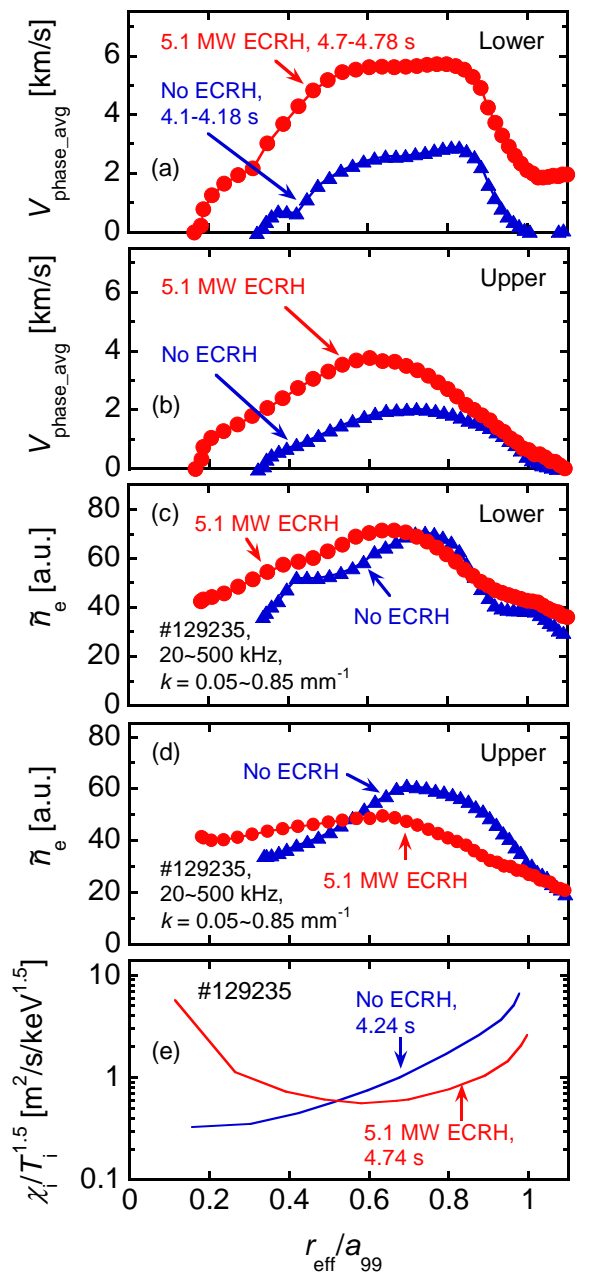

FIG. 11. The radial profiles of (a), (b) the phase velocity, (c), (d) the amplitude of the $n_{\mathrm{e}}$ fluctuation, and (e) $\chi_{\mathrm{i}} / T_{\mathrm{i}}^{1.5}$ before and during the ECRH (5.1 MW). (a) and (c) were obtained at the lower side of the equatorial plane and (b) and (d) were at the upper side. 
was improved at the plasma edge by the on-axis ECRH superposition on the NBI-sustained plasmas although the ion thermal confinement was degraded in the plasma core with increase of $T_{\mathrm{e}} / T_{\mathrm{i}}$. The normalized ion thermal diffusivity $\chi_{\mathrm{i}} / T_{\mathrm{i}}^{1.5}$ at the plasma edge was reduced by $70 \%$. The improvement of the ion thermal confinement at the edge led to increase in $T_{\mathrm{i}}$ in the entire plasma region, especially for higher $n_{\mathrm{e}}$ plasmas, even though the core transport was degraded. The feature is beneficial for a high $T_{\mathrm{i}}$ scenario for high density plasmas under electron heating dominant such as DEMO. The high temperature plasmas obtained in the work was of still low density compared with the reactor $\left(\sim 10^{20} \mathrm{~m}^{-3}\right)$. The confinement characteristics of ITB plasmas with higher density will be investigated in the LHD. Also scenarios of an impurity control have to be established toward DEMO. High temperature plasmas with a low collisionality as presented in the paper are beneficial for the investigation of the impurity control [38].

The structure of the $n_{\mathrm{e}}$ fluctuation at the edge was segmentalized and the amplitude at the upper side of the equatorial plane decreased in the outer half of the plasma. It has not been clarified why the edge ion thermal confinement improved during the on-axis ECRH. The accumulation of the systematic data is necessary. The present data were obtained for the discharges only with the on-axis ECRH superposition. We need to conduct experiments using an off-axis ECRH to clarify the ECRH effect on the edge-core interaction of the ion thermal confinement.

\section{Acknowledgment}

This work was supported by NIFS grants ULRR701, 702, 801, 802, 804, 808, 809, KLER045.

\section{References}

[1] WAGNER F. et al., Phys. Rev. Lett. 49 (1982) 1408.

[2] KOIDE Y. et al., Phys. Rev. Lett. 49 (1994) 3662.

[3] FUJISAWA A., Plasma Phys. Control. Fusion 45 (2003) R1.

[4] FUJITA T. et al., Plasma Phys. Control. Fusion 46 (2004) A35.

[5] SCHMITZ L. et al., Nucl. Fusion 52 (2012) 023003.

[6] SOMMER F. et al., Nucl. Fusion 55 (2015) 033006.

[7] YOSHIDA M. et al., Nucl. Fusion 55 (2015) 073014.

[8] NAGAOKA K., Nucl. Fusion 55 (2015) 113020.

[9] YU D.L. et al., Nucl. Fusion 56 (2016) 056003.

[10] IDA K. et al., Phys. Rev. Lett. 91 (2003) 085003.

[11] TAKEIRI Y. et al., Fusion Sci. Technol. 46 (2004) 106.

[12] SHIMOZUMA T. et al., Nucl. Fusion 45 (2005) 1396.

[13] TAKAHASHI H. et al., Phys. Plasmas 21 (2014) 061506.

[14] YOSHINUMA M. et al., Fusion Sci. Technol. 58 (2010) 375.

[15] IDO T. et al., Rev. Sci. Instrm. 77 (2006) 10 F523.

[16] YOKOYAMA M. et al., Nucl. Fusion 47 (2007) 1213.

[17] KLINGER T. et al., Plasma Phys. Control. Fusion 59 (2017) 014018. 
[18] KANEKO O. et al., Plasma Fusion Research 4 (2009) 027.

[19] IDA K. et al., Nucl. Fusion 49 (2009) 095024.

[20] IDA K. et al., Nucl. Fusion 50 (2010) 064007.

[21] NAGAOKA K. et al., Nucl. Fusion 51 (2011) 083022.

[22] TAKAHASHI H. et al., Nucl. Fusion 53 (2013) 073034.

[23] SAKAMOTO Y. et al., Nucl. Fusion 41 (2001) 865.

[24] IIYOSHI A et al., Nucl. Fusion 39 (1999) 1245.

[25] YAMADA Y for the LHD Experiment Group, Nucl. Fusion 51 (2011) 094021.

[26] TAKEIRI Y. et al., Fusion Sci. Technol. 58 (2010) 482.

[27] OSAKABE M. et al., 2007 Proc. 17th Int. Toki Conf./16th Int. Stellarator/Heliotron Workshop 2007 (Toki, Japan) P2-079 http://www.nifs.ac.jp/itc/itc17/file/PDF proceedings/poster2/P2-079.pdf.

[28] SHIMOZUMA T. et al., Fusion Sci. Technol. 58, (2010) 530.

[29] TAKAHASHI H. et al., Fusion Sci. Technol. 57 (2010) 19.

[30] TSUJIMURA T.Ii et al., Nucl. Fusion 55 (2015) 123019.

[31] LEE H. et al., Plasma Phys. Control. Fusion 55 (2013) 014011.

[32] WAKASA A. et al., Jpn. J. Appl. Phys. 46 (2007) 1157.

[33] INAGAKI S. et al., Phys. Rev. Lett. 107 (2011) 115001.

[34] KOBAYASHI T. et al., Nucl. Fusion 54 (2014) 073017.

[35] TOKUZAWA T. et al., Plasma and Fusion Research 9 (2014) 1402149.

[36] TANAKA K. et al., Rev. Sci. Instrum 79 (2008) 10 E702.

[37] MICHAEL C. et al, Rev. Sci. Instrum. 86, (2015), 093503.

[38] VELASCO J.L. et al., Nucl. Fusion 57 (2017) 016016. 